\title{
Korrespondenzen.
}

Aus der Medizinischen Klinik der Universität in Bologna. (Direktor: Prof. Albertoni.)

\section{Zur Frage der Adrenalinempfindlichkeit des menschlichen} Organismus.

Von Dr. Angelo Sanguinetti, Assistent.

Schon am 30. IV: 1921 habe ich der Zeitschrift "Le malattie del cuore" Folgendes geschrieben: "Die bisherigen Forschungen; um den Zweck des vegetativen Nervensystems festzusetzen (Adrenalinverwendung mittels subkutaner Injektionen) haben den meisten Teil ihres Wertes verloren“. In derselben Arbeit habe ich ferner über die Erfolge, durch die intravenöse Adrenalininjektion die Diagnose des funktionellen $\mathrm{Zu}$ standes des vegetativen Nervensystems zu ermöglichen, berichtet. Die in Nr. 33 veröffentlichte Arbeit von Dr. Csépai enthält dieselben Auffassungen. Ich freue mich, daß Czépai meine Ansichten, von denen er allerdings nichts wußte, bestätigt, möchte aber, daß meine Priorität gesichert bleibt. 